\title{
Sub-barrier capture with quantum diffusion approach
}

\author{
V.V.Sargsyan ${ }^{1}$, R.A.Kuzyakin ${ }^{1}$, G.G.Adamian ${ }^{1, a}$, N.V.Antonenko ${ }^{1}$, W.Scheid ${ }^{2}$, and H.Q.Zhang ${ }^{3}$ \\ 1 Joint Institute for Nuclear Research, 141980 Dubna, Russia \\ 2 Institut für Theoretische Physik der Justus-Liebig-Universität, D-35392 Giessen, Germany \\ 3 China Institute of Atomic Energy, Post Office Box 275, Beijing 102413, China
}

\begin{abstract}
With the quantum diffusion approach the behavior of capture cross sections and mean-square angular momenta of captured systems are revealed in the reactions with deformed and spherical nuclei at sub-barrier energies. With decreasing bombarding energy under the barrier the external turning point of the nucleus-nucleus potential leaves the region of short-range nuclear interaction and action of friction. Because of this change of the regime of interaction, an unexpected enhancement of the capture cross section is found at bombarding energies far below the Coulomb barrier. This effect is shown its worth in the dependence of mean-square angular momentum on the bombarding energy. From the comparison of calculated capture cross sections and experimental capture or fusion cross sections the importance of quasifission near the entrance channel is demonstrated for the actinidebased reactions and reactions with medium-heavy nuclei at extreme sub-barrier energies.
\end{abstract}

\section{Introduction}

The measurement of excitation functions down to the extreme sub-barrier energy region is important for studying the long range behavior of nucleus-nucleus interaction as well as the coupling of relative motion with other degrees of freedom [1-28]. The experimental data obtained are of interest for solving astrophysical problems related to nuclear synthesis. Indications for an enhancement of the $S$-factor, $S=E_{\text {c.m. }} \sigma \exp (2 \pi \eta)[29,30]$, where $\eta\left(E_{\text {c.m. }}\right)=$ $Z_{1} Z_{2} e^{2} \sqrt{\mu /\left(2 \hbar^{2} E_{\mathrm{c} . \mathrm{m} .}\right)}$ is the Sommerfeld parameter, at energies $E_{\text {c.m. }}$. below the Coulomb barrier have been found in Refs. $[10,15,18]$. Its origin is still under discussion.

From the comparison of capture cross sections and fusion cross sections one can show a significant role of the quasifission channel in the reactions with various mediumlight and heavy nuclei at sub-barrier energies. The competition between the complete fusion and quasifission can strongly reduce the value of the fusion cross section and, respectively, the value of the evaporation residue cross section [31-33]. This effect is especially crucial in the production of superheavy nuclei. It worth remembering that first evidences of hindrance for compound nucleus formation in the reactions with massive nuclei at low energies near the Coulomb barrier were observed at GSI already long time ago [34].

To clarify the behavior of capture and fusion cross sections at sub-barrier energies, a further development of the theoretical methods is required [33,35-37]. The conventional coupled-channel approach with realistic set of parameters is not able to describe the fusion cross sections either below or above the Coulomb barrier [18]. The use of a quite shallow nucleus-nucleus potential [38] with an ad-

\footnotetext{
a e-mail: adamian@theor.jinr.ru
}

justed repulsive core considerably improves the agreement between the calculated and experimental data. Besides the coupling with collective excitations, the dissipation, which is simulated by an imaginary potential in Ref. [38] or by damping in each channel in Ref. [39], seems to be important. The quantum diffusion approach based on the quantum master-equation for the reduced density matrix has been suggested in Ref. [40] for the describing capture process. The collisions of nuclei are treated in terms of a single collective variable: the relative distance between the colliding nuclei. Our approach takes into consideration the fluctuation and dissipation effects in collisions of heavy ions which model the coupling with various channels. In the present paper the capture model [40] is applied.

\section{Comparison with experimental data and predictions}

One can see in Figs. 1-4 that with decreasing $E_{\text {c.m. }}$ up to about 3 - $18 \mathrm{MeV}$ below the Coulomb barrier the regime of interaction is changed because at the external turning point the colliding nuclei do not reach the region of nuclear interaction where the friction plays a role. As result, at smaller $E_{\text {c.m. }}$ the capture cross sections $\sigma_{\text {cap }}$ fall with a smaller rate. Therefore, an effect of the change of fall rate of sub-barrier capture cross section should be in the data if we assume that the friction starts to act only when the colliding nuclei approach the barrier. Note that at sub-barrier energies the experimental data have still large uncertainties to make a firm experimental conclusion about this effect. The effect seems to be more pronounced in collisions of spherical nuclei, where the regime of interaction is changed at $E_{\text {c.m. }}$ up to about 3.5 - $5 \mathrm{MeV}$ below the Coulomb barrier [40]. 

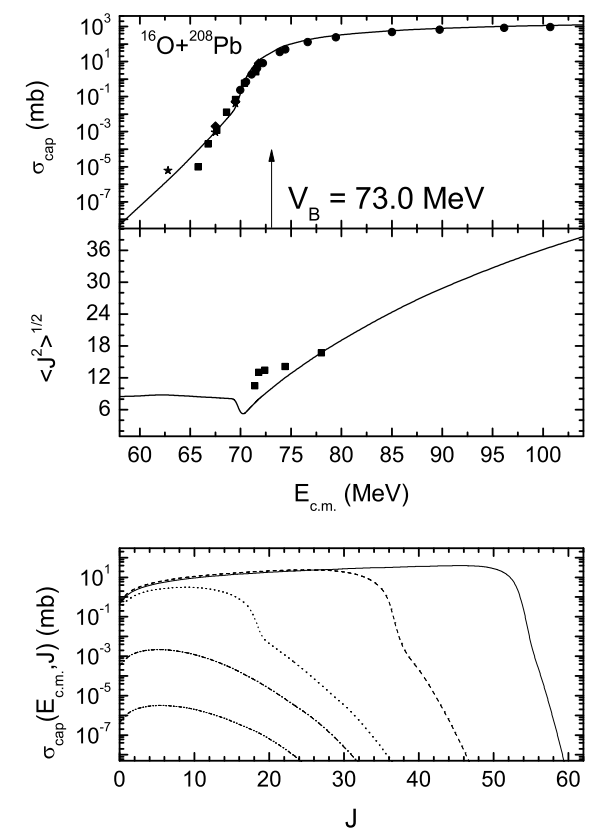

Fig. 1. The calculated (lines) capture cross section (upper part), average angular momenta of captured system (middle part) versus $E_{\text {c.m. }}$, and partial capture cross sections (lower part) versus $J$ at $E_{\text {c.m. }}=65$ (dash-dot-dotted line), 69.5 (dash-dotted line), 73 (dotted line), 83 (dashed line), and 100 (solid line) MeV for the ${ }^{16} \mathrm{O}+{ }^{208} \mathrm{~Pb}$ reaction with the spherical nuclei. The experimental cross sections marked by closed squares, circles, rhombus, stars are from Refs. [18, 8, 11,5], respectively. The experimental values of $\left\langle J^{2}\right\rangle$ (solid squares) are taken from Ref. [41]. The value of the Coulomb barrier $V_{b}$ is indicated by arrow.

The calculated mean-square angular momenta $\left\langle J^{2}\right\rangle$ of captured systems versus $E_{\text {c.m. }}$ are presented in Figs. 1 and 5 for the reactions ${ }^{16} \mathrm{O}+{ }^{208} \mathrm{~Pb}$ and ${ }^{16} \mathrm{O}+{ }^{232} \mathrm{Th},{ }^{238} \mathrm{U}$. At energies below the barrier $\left\langle J^{2}\right\rangle$ has a minimum. This minimum depends on the deformations of nuclei and on the factor $Z_{1} \times Z_{2}$. For the reactions ${ }^{16} \mathrm{O}+{ }^{232} \mathrm{Th},{ }^{238} \mathrm{U}$, these minima are about $7-8 \mathrm{MeV}$ below the corresponding Coulomb barriers, respectively. The experimental data [41] indicate the presence of the minimum as well. On the left-hand side of this minimum the dependence of $\left\langle J^{2}\right\rangle$ on $E_{\text {c.m. }}$ is rather weak. Note that the found behavior of $\left\langle J^{2}\right\rangle$, which is related to the change of the regime of interaction between the colliding nuclei, would affect the angular anisotropy of the products of fission-like fragments following capture. Indeed, the values of $\left\langle J^{2}\right\rangle$ are extracted from data on angular distribution of fission-like fragments [25].

The agreement between the experimental $\left\langle J^{2}\right\rangle$ and those calculated with Wong-type formula is rather bad. At energies below the barrier the $\left\langle J^{2}\right\rangle$ has no a minimum (see Fig. 5). However, for the considered reactions the saturation values of $\left\langle J^{2}\right\rangle$ are close to those obtained in our formalism.

In Fig. 6 the calculated astrophysical $S$-factor versus $E_{\text {c.m. }}$ is shown for the ${ }^{16} \mathrm{O}+{ }^{238} \mathrm{U}$ reaction. The $S$-factor has
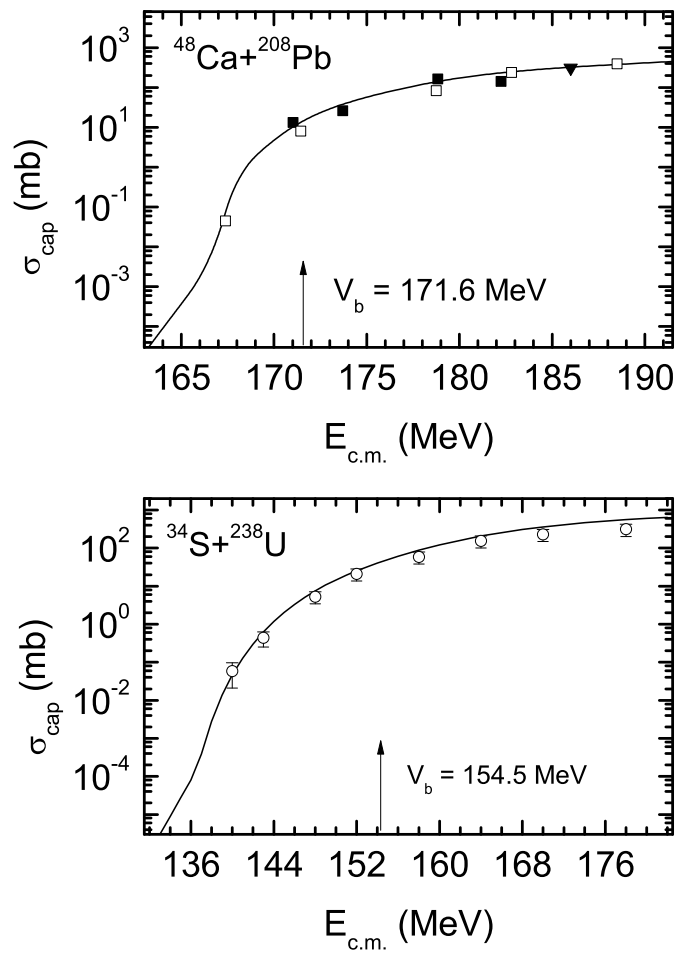

Fig. 2. The calculated (solid lines) capture cross section versus $E_{\text {c.m. }}$ for the reactions ${ }^{48} \mathrm{Ca}+{ }^{208} \mathrm{~Pb}$ (upper part) and ${ }^{34} \mathrm{~S}+{ }^{238} \mathrm{U}$ (lower part). The experimental cross sections are taken from Refs. [42] (closed squares and triangle), [19] (open squares), and [26] (open circles). The value of the Coulomb barrier $V_{b}$ is indicated by arrow. The static quadrupole deformation parameters are: $\beta_{1}\left({ }^{48} \mathrm{Ca}\right)=\beta_{2}\left({ }^{208} \mathrm{~Pb}\right)=0, \beta_{1}\left({ }^{34} \mathrm{~S}\right)=0.125$, and $\beta_{2}\left({ }^{238} \mathrm{U}\right)=0.286$ [43].

a maximum which is seen in experiments [10,15,38]. After this maximum $S$-factor slightly decreases with decreasing $E_{\text {c.m. }}$ and then starts to increase. This effect seems to be more pronounced in collisions of spherical nuclei. The same behavior has been revealed in Refs. [51] by extracting the $S$-factor from the experimental data.

In Fig. 6, the so-called logarithmic derivative, $L\left(E_{\mathrm{c} . \mathrm{m} .}\right)$ $=d\left(\ln \left(E_{\mathrm{c} . \mathrm{m} .} \sigma_{\text {cap }}\right)\right) / d E_{\mathrm{c} . \mathrm{m} .}$, and the barrier distribution $d^{2}\left(E_{\text {c.m. }} \sigma_{\text {cap }}\right) / d E_{\text {c.m. }}^{2}$ are presented for the ${ }^{16} \mathrm{O}+{ }^{238} \mathrm{U}$ reaction. The logarithmic derivative strongly increases below the barrier and then has a maximum at $E_{\text {c.m. }} \approx V_{b}^{\text {orient }}$ (sphere-pole)- $3 \mathrm{MeV}$ (at $E_{\text {c.m. }} \approx V_{b}-3 \mathrm{MeV}$ for the case of spherical nuclei). The maximum of $L$ corresponds to the minimum of the $S$-factor. The barrier distributions calculated with an energy increment $0.2 \mathrm{MeV}$ have only one maximum at $E_{\text {c.m. }} \approx V_{b}^{\text {orient }}$ (sphere-sphere $)=V_{b}$ as in the experiment [50]. With an increasing increment the barrier distribution is shifted to lower energies. Assuming a spherical target nucleus in the calculations, we obtain a more narrow barrier distribution. 

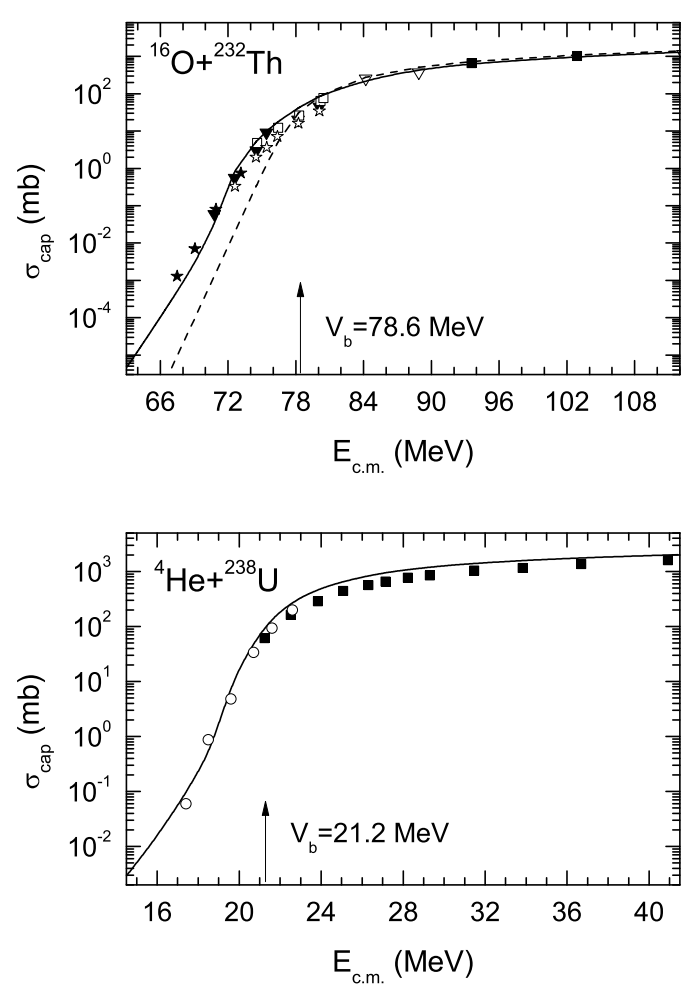

Fig. 3. The same as in Fig. 2, but for the reactions for the reactions ${ }^{16} \mathrm{O}+{ }^{232} \mathrm{Th}$ and ${ }^{4} \mathrm{He}+{ }^{238} \mathrm{U}$. The experimental data in the upper part are taken from Refs. [44] (open triangles), [2] (closed triangles), [45] (open squares), [46] (closed squares), [6] (open stars) and [7] (closed stars). The fission cross sections from Refs. [9] and [47] are shown in the lower part by open circles and solid squares, respectively. The value of the Coulomb barrier $V_{b}$ for the spherical nuclei is indicated by arrow. The dashed curve represents the calculation with the Wong formula [48]. The static quadrupole deformation parameters are: $\beta_{2}\left({ }^{232} \mathrm{Th}\right)=0.261$ $\beta_{2}\left({ }^{238} \mathrm{U}\right)=0.286[43]$ and $\beta_{1}\left({ }^{4} \mathrm{He}\right)=\beta_{1}\left({ }^{16} \mathrm{O}\right)=0$.

\section{Capture cross sections in reactions with large fraction of quasifission}

In the case of large values of $Z_{1} \times Z_{2}$ the quasifission process competes with complete fusion at energies near barrier and can lead to a large hindrance for fusion, thus ruling the probability for producing superheavy elements in the actinide-based reactions $[32,52]$. Since the sum of the fusion cross section $\sigma_{f u s}$, and the quasifission cross section $\sigma_{q f}$ gives the capture cross section,

$$
\sigma_{c a p}=\sigma_{f u s}+\sigma_{q f}
$$

and $\sigma_{f u s} \ll \sigma_{q f}$ for actinide-based reactions ${ }^{48} \mathrm{Ca}+{ }^{232} \mathrm{Th}$, ${ }^{238} \mathrm{U},{ }^{244} \mathrm{Pu},{ }^{246,248} \mathrm{Cm}$ and ${ }^{50} \mathrm{Ti}+{ }^{244} \mathrm{Pu}$ [32], we have

$$
\sigma_{c a p} \approx \sigma_{q f}
$$

In a wide mass-range near the entrance channel, the quasifission events overlap with the products of deep-inelastic collisions and can not be firmly distinguished. Because of this the mass region near the entrance channel is taken out in the experimental analyses in Refs. [53,54].
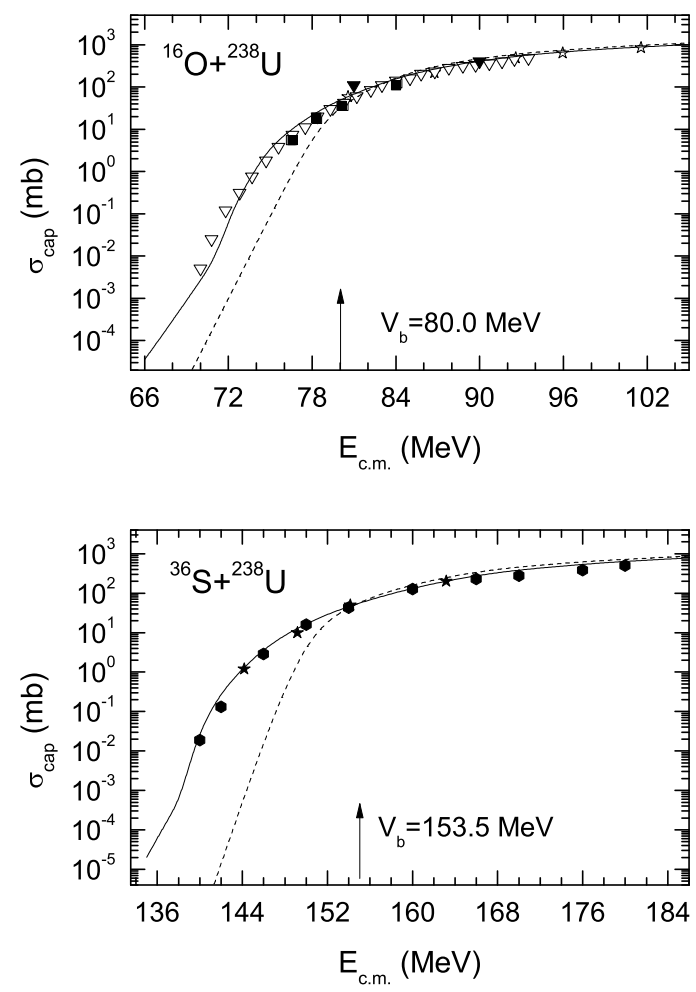

Fig. 4. The same as in Fig. 2, but for the reactions ${ }^{16} \mathrm{O}+$ ${ }^{238} \mathrm{U}$ and ${ }^{36} \mathrm{~S}+{ }^{238} \mathrm{U}$. The experimental cross sections are taken from Refs. [13] (open triangles), [49] (closed triangles), [6] (open squares), [4] (closed squares), [47] (open stars), [24] (closed stars), and [20] (rhombuses). The dashed curve represents the calculation with the Wong formula [48]. The static quadrupole deformation parameters are: $\beta_{2}\left({ }^{238} \mathrm{U}\right)=0.286$ and $\beta_{1}\left({ }^{16} \mathrm{O}\right)=\beta_{1}\left({ }^{36} \mathrm{~S}\right)=0$.

Thus, by comparing the calculated and experimental capture cross sections one can study the importance of quasifission near the entrance channel for the actinide-based reactions leading to superheavy nuclei.

The capture cross sections for the quasifission reactions [53-55] are shown in Figs. 7 and 8. One can observe a large deviations of the experimental data of Refs. [53, 54] from the the calculated results. The possible reason is an underestimation of the quasifission yields measured in these reactions. Thus, the quasifission yields near the entrance channel are important. Note that there are the experimental uncertainties in the bombarding energies.

One can see in Fig. 9 that the experimental and the theoretical cross sections become closer with increasing bombarding energy. This means that with increasing bombarding energy the quasifission yields near the entrance channel mass-region decrease with respect to the quasifission yields in other mass-regions. As seen in Fig. 9, the quasifission yields near the entrance channel mass-region increase with $Z_{1} \times Z_{2}$. 

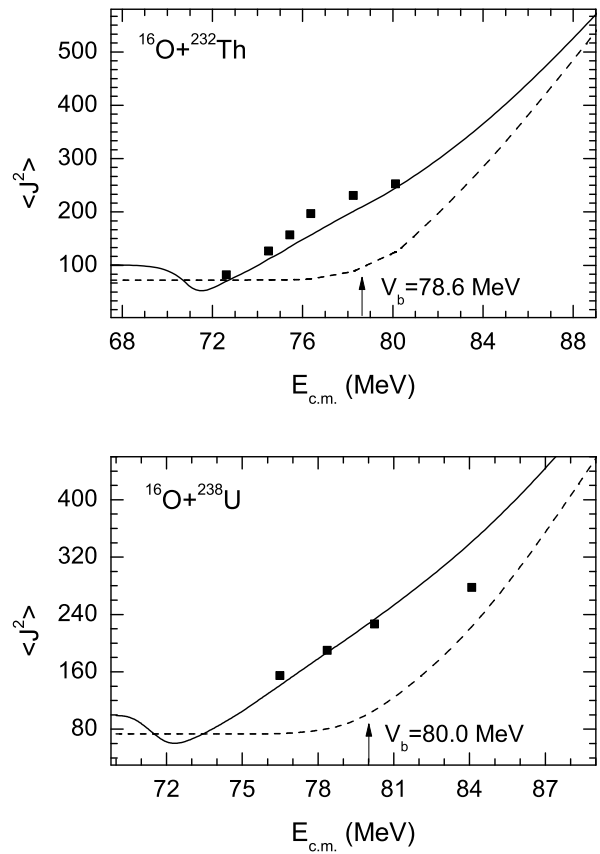

Fig. 5. The calculated mean-square angular momenta versus $E_{\text {c.m. }}$ for the reactions ${ }^{16} \mathrm{O}+{ }^{232} \mathrm{Th},{ }^{238} \mathrm{U}$ are compared with experimental data [6]. The dashed curve represents the calculation by the Wong-type formula [48].

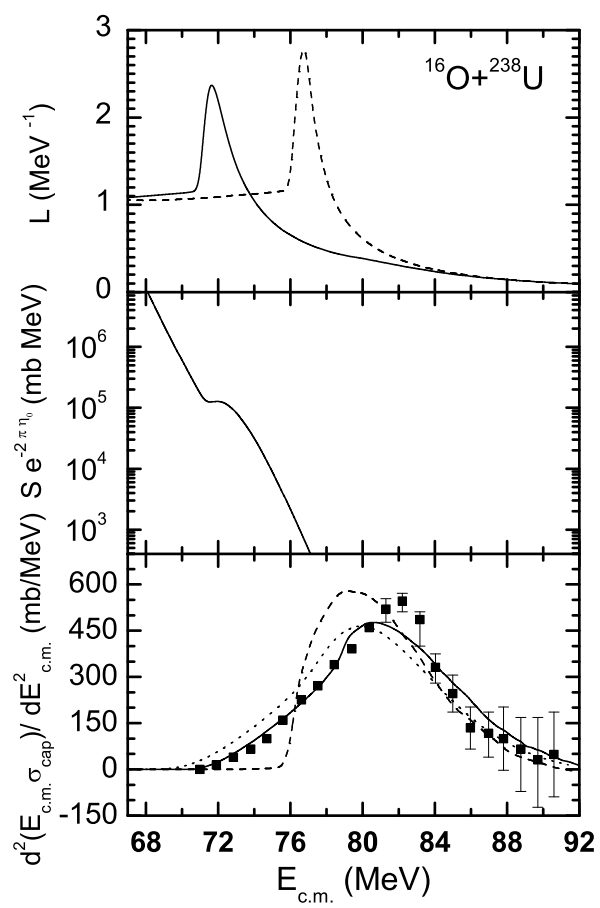

Fig. 6. The calculated values of the astrophysical $S$-factor with $\eta_{0}=\eta\left(E_{\mathrm{c} . \mathrm{m} .}=V_{b}\right)$ (middle part), the logarithmic derivative $L$ (upper part) and the fusion barrier distribution $d^{2}\left(E_{\text {c.m. }} \sigma_{\text {cap }}\right) / d E_{\text {c.m. }}^{2}$. (lower part) for the ${ }^{16} \mathrm{O}+{ }^{238} \mathrm{U}$ reaction. The value of $L$ calculated with the assumption of $\beta_{1}\left({ }^{16} \mathrm{O}\right)=\beta_{2}\left({ }^{238} \mathrm{U}\right)=0$ is shown by a dashed line. The solid and dotted lines show the values of $d^{2}\left(E_{\text {c.m. }} \sigma_{\text {cap }}\right) / d E_{\text {c.m. }}^{2}$ calculated with the increments 0.2 and 1.2 $\mathrm{MeV}$, respectively. The closed squares are the experimental data of Ref. [50].
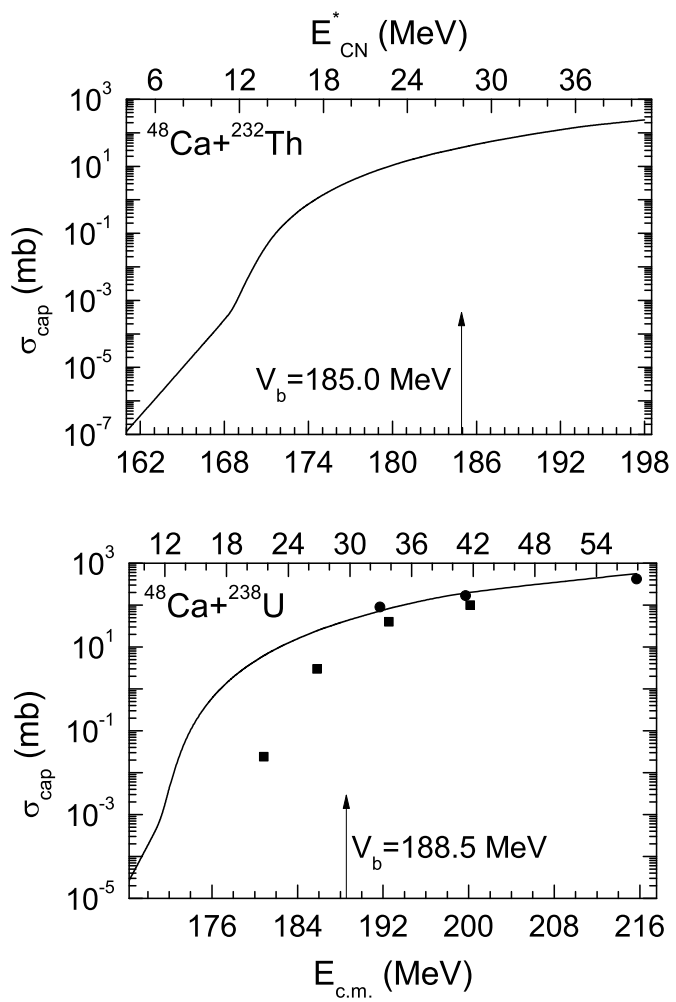

Fig. 7. The same as in Fig. 4, but for the ${ }^{48} \mathrm{Ca}+{ }^{232} \mathrm{Th}^{238} \mathrm{U}$ reactions. The excitation energies $E_{C N}^{*}$ of the corresponding nuclei are indicated. The experimental data are taken from Refs. [53] (marked by squares) and [55] (marked by circles). The static quadrupole deformation parameters are: $\beta_{2}\left({ }^{238} \mathrm{U}\right)=0.286$, $\beta_{2}\left({ }^{232} \mathrm{Th}\right)=0.261$, and $\beta_{1}\left({ }^{48} \mathrm{Ca}\right)=0$.

\section{Origin of fusion hindrance in reactions with medium-mass nuclei at sub-barrier energies}

In Figs. 10 and 11 the calculated capture cross section are presented for the reactions ${ }^{36} \mathrm{~S}+{ }^{48} \mathrm{Ca},{ }^{64} \mathrm{Ni}$ and ${ }^{64} \mathrm{Ni}+$ ${ }^{64} \mathrm{Ni}$. The values of $V_{b}$ are adjusted to the experimental data for the fusion cross sections shown as well. For the systems mentioned above, the difference between the subbarrier capture and fusion cross sections becomes larger with decreasing bombarding energy $E_{\text {c.m. }}$. The same effect one can see for the ${ }^{16} \mathrm{O}+{ }^{208} \mathrm{~Pb}$ reaction. Assuming that the estimated capture and the measured fusion cross sections are correct, the small fusion cross section at energies well below the Coulomb barrier may indicate that other reaction channel is open and the system evolves by other reaction mechanism after the capture. The observed hindrance factor may be understood in term of quasifission whose cross section should be added to the one of fusion to obtain a meaningful comparison with the calculated capture cross section. The quasifission event corresponds to the formation of a nuclear-molecular state or dinuclear system with small excitation energy that separates (in the competition with the compound nucleus formation process) by the quantal tunneling through the Coulomb barrier in a binary event with mass and charge close to the 


\section{FUSION11}
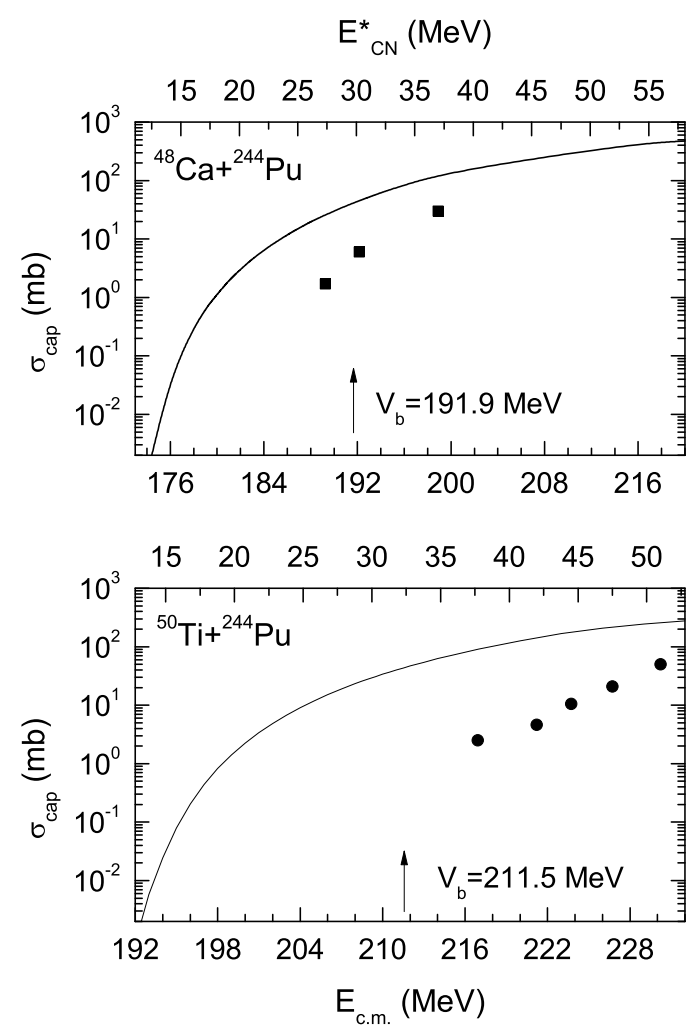

Fig. 8. The same as in Fig. 7 , but for the indicated ${ }^{48} \mathrm{Ca},{ }^{50} \mathrm{Ti}$ $+{ }^{244} \mathrm{Pu}$ reactions. The experimental data are from Refs. [54] (squares) and [53] (circles). The static quadrupole deformation parameters are: $\beta_{2}\left({ }^{244} \mathrm{Pu}\right)=0.293$, and $\beta_{1}\left({ }^{48} \mathrm{Ca}\right)=\beta_{1}\left({ }^{50} \mathrm{Ti}\right)=0$.

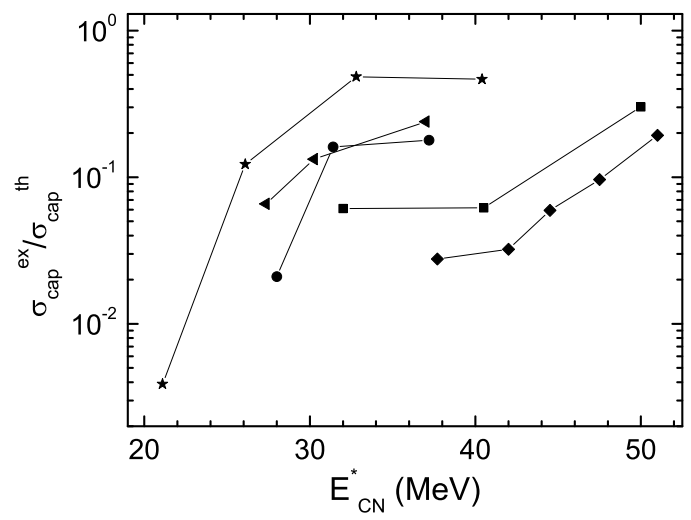

Fig. 9. The ratio of theoretical and experimental capture cross sections versus the excitation energy $E_{\text {c.m. }}$ of the compound nucleus for the reactions ${ }^{48} \mathrm{Ca}+{ }^{238} \mathrm{U}$ (closed stars), ${ }^{48} \mathrm{Ca}+{ }^{244} \mathrm{Pu}$ (closed triangles), ${ }^{48} \mathrm{Ca}+{ }^{246} \mathrm{Cm}$ (closed squares), ${ }^{48} \mathrm{Ca}+{ }^{248} \mathrm{Cm}$ (closed circles), and ${ }^{50} \mathrm{Ti}+{ }^{244} \mathrm{Pu}$ (closed rhombuses).

entrance channel. In this sense the quasifission is the general phenomenon which takes place in the reactions with the massive [31-34], medium-mass and, probably, light nuclei. For the medium-mass and light nuclei, this reaction mode has to be studied in the future experiments: from the mass (charge) distribution measurements one can show the distinct components due to quasifission. The low-energy experimental data would probably provide straight infor-
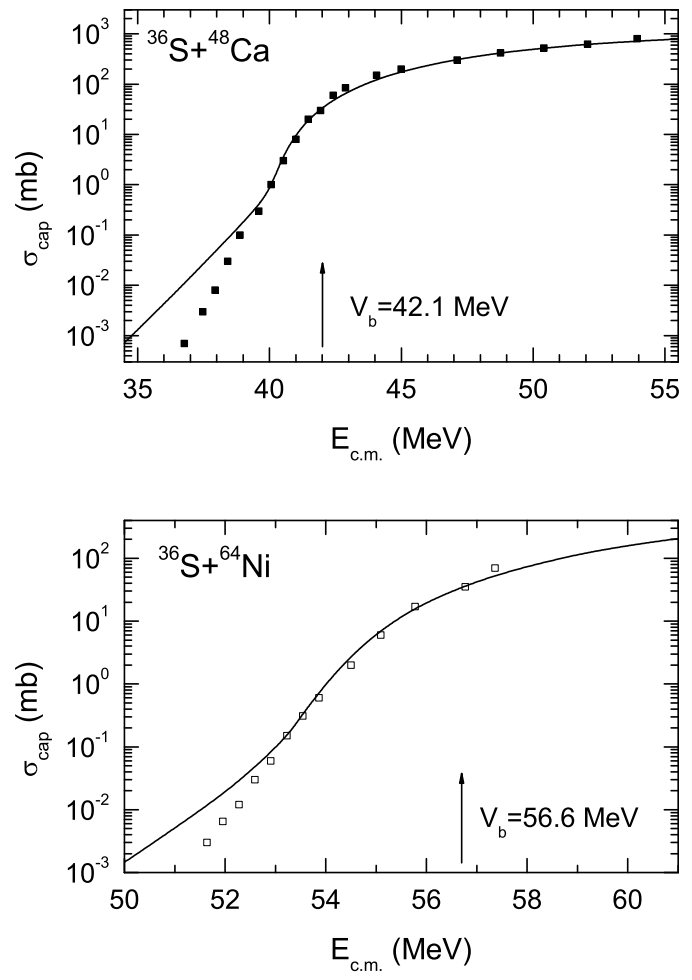

Fig. 10. The calculated capture cross sections versus $E_{\text {c.m. }}$ for the indicated reactions. The experimental fusion cross sections marked by closed and open squares are taken from Refs. [21, 28], respectively. The values of the Coulomb barrier are indicated by arrows. The static quadrupole deformation parameters are: $\beta_{1}\left({ }^{36} \mathrm{~S}\right)=\beta_{2}\left({ }^{48} \mathrm{Ca}\right)=0$ and $\beta_{2}\left({ }^{64} \mathrm{Ni}\right)=0.087$ [56].

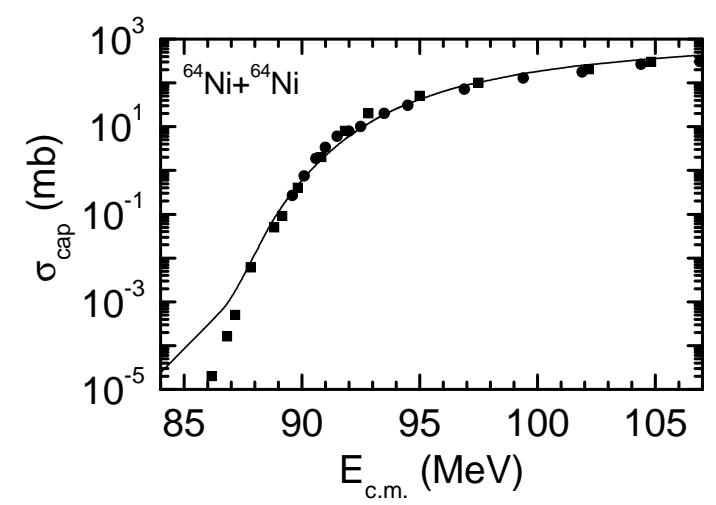

Fig. 11. The calculated capture cross sections versus $E_{\text {c.m. }}$ for the indicated reaction. The experimental fusion cross sections marked by closed circles and squares are taken from Refs. [1, 14], respectively. Here, $\beta_{1,2}\left({ }^{64} \mathrm{Ni}\right)=0.087$.

mation since the high-energy data may be shaded by competing reaction processes such as quasifission and deepinelastic collisions. Note that the binary decay events were already observed experimentally in [57] for the ${ }^{58} \mathrm{Ni}+{ }^{124} \mathrm{Sn}$ reaction at energies below the Coulomb barrier but assumed to be related to deep-inelastic scattering. 


\section{Summary}

The quantum diffusion approach is applied to study the capture process in the reactions with deformed and spherical nuclei at sub-barrier energies. Due to a change of the regime of interaction (the turning-off of the nuclear forces and friction) at sub-barrier energies, the curve related to the capture cross section as a function of bombarding energy has smaller slope. This change is also reflected in the functions $\left\langle J^{2}\right\rangle, L\left(E_{\mathrm{c} . \mathrm{m} .}\right)$, and $S\left(E_{\mathrm{c.m}}\right)$. The mean-square angular momentum of captured system versus $E_{\text {c.m. }}$. has a minimum and then saturates at sub-barrier energies. This behavior of $\left\langle J^{2}\right\rangle$ would increase the expected anisotropy of the angular distribution of the products of fission and quasifission following capture. The astrophysical factor has a maximum and a minimum at energies below the barrier. The maximum of $L$-factor corresponds to the minimum of the $S$ factor. The importance of quasifission near the entrance channel is shown for the actinide-based reactions at near barrier energies and reactions with medium-mass nuclei at extreme sub-barrier energies. One can suggest the experiments to check these predictions.

We thank H. Jia and D. Lacroix for fruitful discussions and suggestions. We are grateful to K. Nishio for providing us his experimental data. This work was supported by DFG, NSFC, and RFBR. The IN2P3-JINR and PolishJINR Cooperation programs are gratefully acknowledged.

\section{References}

1. M.Beckerman et al., Phys.Rev.C 25, (1982) 837.

2. H.Q.Zhang et al., Phys.Rev.C 42, (1990) 1086.

3. F.Scarlassara et al., Z.Phys.A 338, (1991) 171.

4. H.Q.Zhang et al., Phys.Rev.C 49, (1994) 926.

5. Yu.Ts.Oganessian et al., JINR Rapid Communications 75, (1996) 123.

6. Z.Liu et al., Phys.Rev.C 54, (1996) 761.

7. D.M.Nadkarni et al., Phys.Rev.C 59, (1999) R580.

8. C.R.Morton et al., Phys.Rev.C 60, (1999) 044608.

9. M.Trotta et al., Phys.Rev.Lett. 84, (2000) 2342.

10. C.L.Jiang et al., Phys.Rev.Lett. 89, (2002) 052701.

11. S.P.Tretyakova et al., Nucl.Phys.A 734, (2004) E33;

S.P.Tretyakova et al., Nucl.Phys.A 738, (2004) 487.

12. M.Dasgupta and D.J.Hinde, Nucl.Phys.A 734, (2004) 148.

13. K.Nishio et al., Phys.Rev.Lett. 93, (2004) 162701.

14. C.L.Jiang et al., Phys.Rev.Lett. 93, (2004) 012701.

15. C.L.Jiang et al., Phys.Rev.C 71, (2005) 044613.

16. K.Nishio et al., Eur.Phys.J.A 29, (2006) 281.

17. W.Loveland et al., Phys.Rev.C 74, (2006) 044604;

A.M.Vinodkumar et al., Phys.Rev.C 78, (2008) 054608. 18. M.Dasgupta et al., Phys.Rev.Lett. 99, (2007) 192701.

19. E.Prokhorova et al., Nucl.Phys.A 802, (2008) 45.

20. K.Nishio et al., Phys.Rev.C. 77, (2008) 064607.

21. A.M.Stefanini et al., Phys.Rev.C 78, (2008) 044607.

22. D.J.Hinde et al., Phys.Rev.Lett. 101, (2008) 092701.

23. A.Shrivastava et al., Phys.Rev.Lett. 103, (2009) 232702 .
24. M.G.Itkis et al., Nucl.Phys.A 834, (2010) 374c.

25. H.Q.Zhang et al., Phys.Rev.C 81, (2010) 034611.

26. K.Nishio et al., Phys.Rev.C 82, (2010) 024611.

27. C.Signorini et al., Eur.Phys.J.A 44, (2010) 63.

28. G.Montagnoli et al., Nucl.Phys.A 834, (2010) 159c; Phys.Rev.C (2010) 82064609.

29. K.Langanke and C.A.Barnes, Adv.Nucl.Phys. 22, (1996) 173.

30. A.Aprahamian, K.Langanke, and M.Wiescher, Prog.Part.Nucl.Phys. 54, (2005) 535.

31. V.V.Volkov, Particles and Nuclei, 35, (2004) 797.

32. G.G.Adamian, N.V.Antonenko, and W.Scheid, Phys.Rev.C 68, (2003) 034601.

33. G.Giardina et al., Nucl.Phys.A 671, (2000) 165; A.Nasirov et al., Nucl.Phys.A 759, (2005) 342.

34. J.G.Keller et al., Nucl. Phys.A 452, (1986) 173.

35. A.B.Balantekin and N.Takigawa, Rev.Mod.Phys. 70, (1998) 77; L.E.Canto, P.R.S.Gomes, R.Donangelo, and M.S.Hussein, Phys.Rep. 424, (2006) 1.

36. V.V.Sargsyan et al., Phys. Rev. C 80, (2009) 034606; ibid 80, (2009) 047603.

37. G.Hupin and D.Lacroix Phys. Rev.C 81, (2010) 014609; S.Ayik, B.Yilmaz, and D.Lacroix, Phys. Rev.C 81, (2010) 034605.

38. H.Esbensen and C.L.Jiang, Phys. Rev.C 79, (2009) 064619; S.Misicu and H.Esbensen H, Phys. Rev.C 75, (2007) 034606; H.Esbensen and S.Misicu, Phys. Rev. C 76, (2007) 054609.

39. K. Hagino and N.Rowley, AIP Conf. Proc. 1098, (2009) 18.

40. V.V.Sargsyan et al., Eur.Phys.J.A 45, (2010) 125; ibid 47, (2011) 38.

41. R.Vandenbosch, Annu.Rev.Nucl.Part.Sci. 42, (1992) 447.

42. A.J.Pacheco et al., Phys.Rev.C 45, (1992) 2861; R.Bock et al., Nucl.Phys.A 388, (1982) 334.

43. S.Raman, C.W.Nestor, Jr, and Tikkanen P, At.Data Nucl.Data Tables 78, (2001) 1.

44. B.B.Back et al., Phys.Rev.C 32, (1985) 195.

45. T.Murakami et al., Phys.Rev.C 34, (1986) 1353.

46. S.Kailas et al., Phys.Rev.C 59, (1999) 2580.

47. V.E.Viola, Jr and T.Sikkeland, Phys.Rev. 128, (1962) 767.

48. C.Y.Wong, Phys.Rev.Lett. 31, (1973) 766.

49. J.Toke et al., Nucl.Phys.A 440, (1985) 327.

50. D.J.Hinde et al., Phys.Rev.Lett. 74, (1995) 1295.

51. K.Langanke and S.E.Koonin, Nucl.Phys.A 410, (1983) 334; A.Redder et al., Nucl.Phys.A 462, (1987) 385.

52. M.Trotta et al., Eur.Phys.J.A 25, (2005) 615.

53. M.G.Itkis et al., Nucl.Phys.A 787, (2007) 150c.

54. M.G.Itkis et al., Nucl.Phys.A 734, (2004) 136c.

55. W.Q.Shen et al., Phys.Rev.C 36, (1987) 115.

56. P.Möller et al., At.Data Nucl.Data Tables 59, (1995) 185.

57. F.L.H.Wolfs, W.Henning, K.E.Rehm, and J.P.Schiffer, Phys.Lett.B 196, (1987) 113. 